\title{
Utilidad de los índices de oxigenación en pacientes adultos y pediátricos con insuficiencia respiratoria. Revisión narrativa
}

\author{
Adriana Sofía Valero Ortiz ${ }^{1}$ (D), Sandra Patricia Corredor Gamba ${ }^{1}$ (D) , \\ Clara Lizeth Palencia Mojica² (D), Nubia Yalile Castro Chaparro ${ }^{3}$ (D)
}

\section{RESUMEN}

Introducción: los índices de oxigenación son indispensables para evaluar el estado de oxigenación, así como para diagnosticar y clasificar la insuficiencia respiratoria.

Objetivo: analizar la literatura relacionada con la utilidad de los índices de oxigenación Kirby y de saturación en pacientes adultos y pediátricos en unidades de cuidado intensivo con insuficiencia respiratoria.

Método: revisión bibliográfica disponible en las bases de datos Science Direct, Scopus, OVID, Medline y Proquest. Revisión de 20 tesis alineadas con literatura gris, con la ventana de observación 2010-2020. Para seleccionar la información se aplicó la guía Critical Appraisal Skills Programme en español. Se incluyeron 47 artículos originales y 9 tesis, que superaron el $75 \%$ de los criterios evaluados. Se excluyeron 30 estudios correspondientes a revisiones de tema y de caso, así como 10 tesis de pregrado en licenciaturas relacionadas con el cuidado de la salud.

Resultados: la utilidad de los índices de oxigenación en insuficiencia respiratoria en pacientes adultos y pediátricos en cuidado intensivo corresponde a la correlación entre el índice de Kirby y la relación entre saturación parcial de oxígeno y la fracción inspirada de oxígeno como marcador de oxigenación, seguido de la utilización de estos para predicción de mortalidad. En menor proporción, establecen el fracaso de la ventilación mecánica y un diagnóstico temprano del síndrome de dificultad respiratoria.

Conclusiones: según los estudios, el índice de saturación se usa como sustituto en la evaluación de la oxigenación, gravedad de la patología y marcador pronóstico de mortalidad en insuficiencia respiratoria tanto en pacientes adultos como en pediátricos.

Palabras clave: oxigenación; insuficiencia respiratoria; cuidado intensivo; índice; mortalidad.

1 Universidad de Boyacá (Tunja, Colombia).

2 Fundación Universitaria Iberoamericana FUNIBER (Bogotá, Colombia).

${ }^{3}$ Hospital Universitario San Rafael de Tunja (Colombia).

Autora de correspondencia: Adriana Sofía Valero Ortiz. Correo electrónico: svalero9@uniboyaca.edu.co Citar este artículo así:

Valero Ortiz AS, Corredor Gamba SP, Palencia Mojica CL, Castro Chaparro NY. Utilidad de los índices de oxigenación en pacientes adultos y pediátricos con insuficiencia respiratoria: revisión narrativa. Rev Investig Salud Univ Boyacá. 2020;7(2):173-194. doi: https://doi. org/10.24267/23897325.517 


\title{
Usefulness of oxygenation indices in adult and pediatric patients with respiratory failure. Narrative review
}

\begin{abstract}
Introduction: Indispensable oxygenation indices to evaluate oxygenation status, diagnosis and classification of respiratory failure.

Objective: To analyze the literature related to the usefulness of Kirby oxygenation and saturation indices in adult and pediatric patients in intensive care units with respiratory failure.

Method: Bibliographic review available in the databases Science Direct, Scopus, OVID, Medline, and Proquest, review of 20 theses aligned to gray literature, with observation window 2010-2020. For the selection of the information the Spanish Critical Appraisal Skills Programme guide was applied. Forty-seven original articles and 9 theses were included, exceeding $75 \%$ of the evaluated criteria; 30 studies corresponding to reviews of topics and case reviews; also 10 undergraduate theses in health care related degrees were excluded.
\end{abstract}

Results: The usefulness of the oxygenation indexes in respiratory failure in adult and pediatric patients in intensive care corresponds to the correlation between the Kirby index and the relationship between partial oxygen saturation and the inspired oxygen fraction as a marker of oxygenation, followed by the use of these for predicting mortality. To a lesser extent they establish failure of mechanical ventilation and early diagnosis of respiratory distress syndrome.

Conclusions: According to the studies, the saturation index is used as a substitute in the evaluation of oxygenation, severity of the pathology and prognostic marker of mortality in respiratory failure in both adult and pediatric patients.

Keywords: oxygenation; respiratory failure; intensive care; index; mortality. 


\title{
Utilidade dos índices de oxigenação em pacientes adultos e pediátricos com insuficiência respi-ratória. Revisão narrativa
}

\begin{abstract}
RESUMO
Introdução: os índices de oxigenação são essenciais para avaliar o estado da oxigenação, bem como para diagnosticar e classificar a insuficiência respiratória.

Objetivo: analisar a literatura relacionada à utilidade dos índices de oxigenação e saturação de Kirby em pacientes adultos e pediátricos internados em unidades de terapia intensiva com insuficiência respiratória.

Método: revisão bibliográfica dispo-nível nas bases de dados Science Direct, Scopus, OVID, Medline e Proquest. Revisão de 20 teses alinhadas com a literatura cinza, com a janela de observação 20102020. Para selecionar as informa-ções, foi aplicado o guia do Critical Appraisal Skills Programme em espanhol. Foram incluídos 47 artigos e 9 teses, que ultrapassaram $75 \%$ dos critérios avaliados. Foram excluídos 30 estudos corres-pondentes a revisões de temas e casos, além de 10 teses de graduação em cursos relacionadas à saúde.

Resultados: a utilidade dos índices de oxigenação na insuficiência respiratória em pacientes adultos e pediátricos em terapia intensiva corresponde à correlação entre o índice de Kirby e a relação entre a saturação parcial de oxigênio e a fração inspirada de oxigênio como marcador de oxigenação, seguida do uso de estes para previsão de mortalidade. Em menor grau, eles estabelecem a falha da ventilação mecânica e um diagnóstico precoce da síndrome do desconforto respiratório.
\end{abstract}

Conclusões: de acordo com os estudos, o índice de saturação é utilizado como substituto na avaliação da oxigenação, gravi-dade da patologia e marcador prognóstico de mortalidade por insuficiência respiratória em pacientes adultos e pediátricos.

Palavras-chave: oxigenação; insuficiência respiratória; cuidado intensivo; índice; mortalidade. 


\section{INTRODUCCIÓN}

Para evaluar el estado de oxigenación de los pacientes que se encuentran en estado crítico con insuficiencia respiratoria se cuenta con los índices de oxigenación, los cuales permiten conocer el diagnóstico, el seguimiento, el grado de severidad de la insuficiencia y el manejo clínico integral de los pacientes hospitalizados en las unidades de cuidado intensivo adulto y pediátrico.

Clínicamente, dos pruebas funcionales establecen el estado de oxigenación en un individuo: la primera es la gasometría arterial, una prueba invasiva que determina la presión arterial de oxígeno $\left(\mathrm{PaO}_{2}\right)$ y que al relacionarla con la fracción inspirada de oxígeno $\left(\mathrm{FiO}_{2}\right)$, permite determinar el grado de hipoxemia; la segunda es la oximetría de pulso, que no es invasiva y censa el porcentaje de saturación de oxígeno $\left(\mathrm{SpO}_{2}\right)$, y al relacionarla con la $\mathrm{FiO}_{2}\left(\mathrm{SpO}_{2} / \mathrm{FiO}_{2}\right)$, evalúa la oxigenación del paciente. Según la revisión del estado de arte, en la última década se ha incrementado la utilización de la relación $\mathrm{PaO}_{2} / \mathrm{FiO}_{2}$ y la relación porcentaje de $\mathrm{SpO}_{2} / \mathrm{FiO}_{2}$ para analizar la función pulmonar, ya que se consideran uno de los índices usados de manera rutinaria en la evaluación clínica del paciente crítico $(1,2)$.

Los índices de oxigenación más utilizados en cuidado intensivo adulto y pediátrico son: Kirby $\left(\mathrm{PaO}_{2} / \mathrm{FiO}_{2}\right)$, índice de oxigenación e índice de saturación, el cual corresponde a la relación $\mathrm{SpO}_{2} /$ $\mathrm{FiO}_{2}$, que se han estudiado como predictores de hipoxemia, fracaso en la extubación, ventilación mecánica y predicción de mortalidad en pacientes con síndrome de dificultad respiratoria del adulto (SDRA) (3-6); además, como parámetros para evaluar la oxigenación, optimizar el soporte ventilatorio mecánico y establecer un pronóstico de supervivencia (7-11).

Desde el punto de vista fisiológico, en el paciente críticamente enfermo se evidencian alteraciones ventilatorias y de oxigenación que influyen en su morbimortalidad, lo cual debe tratarse de forma rápida con estrategias terapéuticas del cuidado respiratorio, como oxigenoterapia de bajo y alto flujo, aerosolterapia y soporte ventilatorio no invasivo e invasivo, si está indicado, de acuerdo con la severidad de la insuficiencia respiratoria. La estancia hospitalaria prolongada, al igual que el soporte ventilatorio invasivo, genera alteraciones en la mecánica respiratoria, con aumento del trabajo de los músculos, incremento del consumo de oxígeno y, por ende, del trabajo respiratorio, lo que favorece la fatiga muscular y, con ello, el destete fallido $(12,13)$.

En función de lo señalado, se consideró relevante llevar a cabo una revisión narrativa de la literatura sobre los estudios que se han elaborado desde el enfoque clínico, en las áreas de cuidado intensivo adulto y pediátrico, en los últimos 10 años, con 
énfasis en la utilidad de estos índices para evaluar el estado de oxigenación de los pacientes ingresados, así como para evidenciar que se ha utilizado más el índice $\mathrm{PaO}_{2} / \mathrm{FiO}_{2}$ en el manejo de la insuficiencia y que en menor frecuencia de uso se encuentra el índice de saturación $\mathrm{SpO}_{2} / \mathrm{FiO}_{2}$, dado que la mayoría de estudios refiere que existe una asociación lineal entre estos dos; además de considerarse útiles como predictores de mortalidad, extubación, gravedad y pronóstico en insuficiencia respiratoria.

De esta manera, la evaluación de la oxigenación a través del índice de saturación $\mathrm{SpO}_{2} / \mathrm{FiO}_{2}$ como parámetro no invasivo refleja de forma rápida el grado de hipoxemia indirecta y permite clasificar la severidad de la oxigenación en trastornos pulmonares agudos (14).

De acuerdo con lo descrito, el objetivo de esta revisión narrativa se centró en el análisis de la evidencia clínica relacionada con la utilidad de utilidad de los índices de oxigenación Kirby y de saturación en pacientes adultos y pediátricos en unidades de cuidado intensivo con insuficiencia respiratoria.

\section{MATERIALES Y MÉTODOS}

Desde una perspectiva narrativa y crítica, se indagó en ocho bases de datos internacionales: Science Direct, Scopus, OVID, Medline, Proquest,
Pubmed, SciELO y Redalyc. Cuatro investigadores con experiencia clínica del cuidado crítico adulto y pediátrico llevaron a cabo la revisión de 80 artículos publicados, además de 20 tesis de doctorado, maestría o especialidad en cuidado crítico y neumología (considerada como literatura gris), con una ventana de tiempo comprendida entre 2010 y 2020, en tres etapas. La primera se realizó en dos meses, efectuando una búsqueda sistemática de los estudios y aplicando la estrategia de búsqueda estandarizada y aplicable a todos los índices: "fallo respiratorio", "índices de oxigenación" y "cuidado intensivo", incluidos en el MeSH, DeCs y conectores. De igual manera, se recurrió a la búsqueda de descriptores no controlados, es decir, de términos que aun cuando no están indexados en las bases anteriores, son relevantes para la identificación de estudios. Entre estos se incluyeron: $\mathrm{SpO}_{2} / \mathrm{FiO}_{2}, \mathrm{PaO}_{2} / \mathrm{FiO}_{2}$, predictores, mortalidad y correlación.

La segunda etapa, con una duración de un mes, estuvo constituida por la selección de los estudios, aplicando la guía en español propuesta por la Critical Appraisal Skills Programme, según los siguientes criterios: 1) que refirieran de manera clara el enfoque clínico de los índices de oxigenación en pacientes adultos y pediátricos, 2) que reportaran resultados aplicados en pacientes adultos y pediátricos en unidades de cuidado intensivo 3) que especificaran de manera clara la utilidad de los índices de oxigenación en la 
insuficiencia respiratoria y 4) que hubieran sido publicados en los últimos 10 años. Una vez aplicada la guía, se sistematizó la información de acuerdo con las categorías de análisis tratadas en los artículos.

De la segunda etapa resultaron 56 documentos de interés en la temática, que superaron el $75 \%$ de los criterios evaluados con la lista de verificación (figura 1): título, autores, año de publicación, tipo de estudio, revista, objetivos, metodología, variables y conclusiones, y generaron, así, la tercera etapa, con duración de tres meses, donde se analizaron cuatro apartados principales: 1) ca- racterización de las publicaciones, 2) utilidad de la $\mathrm{PaO}_{2} / \mathrm{FiO}_{2}$ y $\mathrm{SpO}_{2} / \mathrm{FiO}_{2}$ en pacientes pediátricos en cuidado intensivo, 3) utilidad de la $\mathrm{PaO}_{2} / \mathrm{FiO}_{2}$ y $\mathrm{SpO}_{2} / \mathrm{FiO}_{2}$ en pacientes adultos en cuidado intensivo y 4) la $\mathrm{PaO}_{2} / \mathrm{FiO}_{2}$ y la $\mathrm{SpO}_{2} / \mathrm{FiO}_{2}$ como predictores de mortalidad en cuidado intensivo. De los 56 documentos revisados, según el enfoque de investigación, la mayoría correspondieron a prospectivos transversales y longitudinales descriptivos, además de la literatura gris, los cuales soportaron la revisión narrativa y evidenciaron la utilidad de estos índices de oxigenación en la población adulta y pediátrica con insuficiencia respiratoria.

Figura 1. Artículos seleccionados para la revisión narrativa

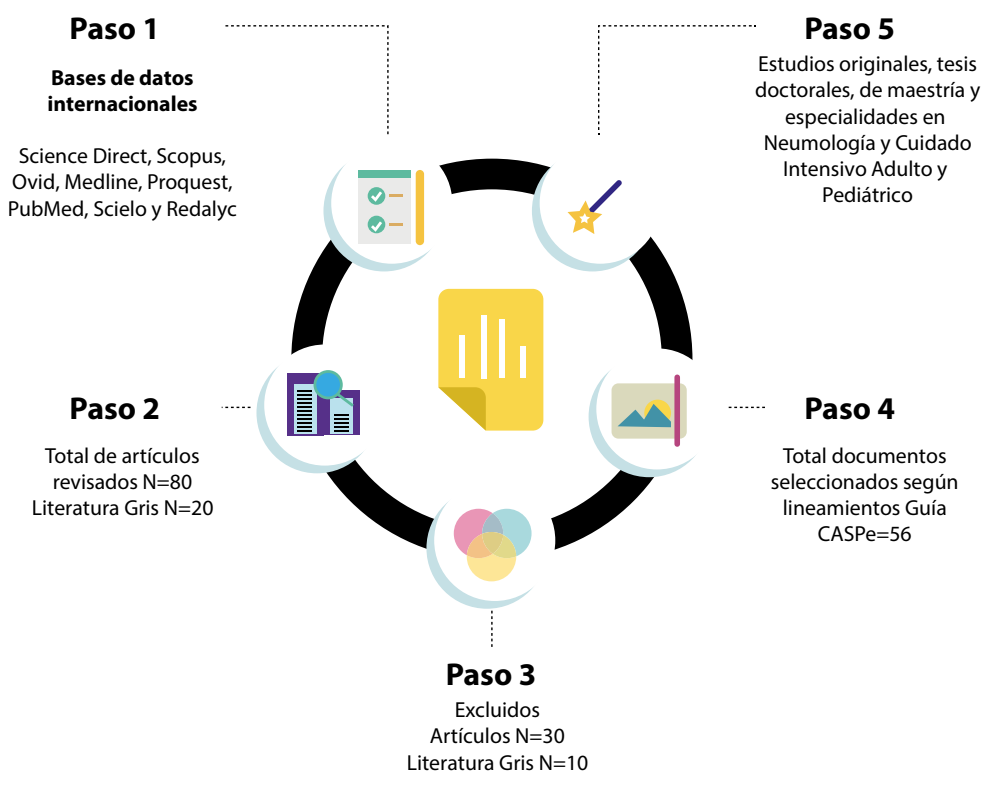




\section{RESULTADOS Y DISCUSIÓN}

Una vez efectuada la búsqueda en las bases de datos, se analizaron 80 artículos y 20 documentos de literatura gris, de los cuales 56 documentos cumplieron con los criterios evaluados.

Entre los países de mayor producción científica en el tema, se destacan Estados Unidos, Japón, Cuba, España, India, Ecuador, Guatemala y Colombia. Según la clasificación internacional y nacional de revistas homologadas según MinCiencias, 32 se enfocan en el ámbito del cuidado intensivo adulto, y 18, en el área pediátrica.

En el contexto clínico, la monitoría ventilatoria utiliza los índices de oxigenación, y uno de los más utilizados es el índice de Kirby $\left(\mathrm{PaO}_{2} / \mathrm{FiO}_{2}\right)$, también útil en la predicción de hipoxemia en disfunción pulmonar aguda. Así mismo, actualmente se ha incrementado la utilidad del índice de saturación $\left(\mathrm{SpO}_{2} / \mathrm{FiO}_{2}\right)$, el cual permite monitorear la oxigenación de manera indirecta y no invasiva. Una vez ejecutada la revisión, se evidenció que la mayoría de los estudios se han desarrollo en los pacientes adultos y pediátricos en cuidado intensivo, por lo cual su uso se hace evidente en la evaluación del estado de oxigenación y como predictor de mortalidad en estos pacientes. A continuación, se presenta la descripción de los resultados de acuerdo con el análisis generado.

\section{Utilidad de la $\mathrm{PaO}_{2} / \mathrm{FiO}_{2}$ y $\mathrm{SpO}_{2} / \mathrm{FiO}_{2}$ en pacientes pediátricos en cuidado intensivo}

De acuerdo con la revisión de la literatura, frente al uso de la $\mathrm{PaO}_{2} / \mathrm{FiO}_{2}$ y $\mathrm{SpO}_{2} / \mathrm{FiO}_{2}$, se evidencia su utilidad para establecer el estado de oxigenación, la mortalidad y la extubación exitosa en pacientes pediátricos con insuficiencia respiratoria, la cual es más susceptible de presentación en este tipo de pacientes, dadas las diferencias fisiológicas con los adultos, representadas en menor reserva metabólica, menor respuesta a la hipoxemia e hipercapnia y menor diámetro de la vía aérea. Ello genera mayor aumento de la resistencia y diferencias en el desarrollo muscular, con menor número de fibras musculares tipo I que llevan a mayor fatiga. De esta manera, el uso de este tipo de índices para establecer el diagnóstico y el manejo terapéutico precoz se consideran medidas fundamentales para prevenir o tratar la insuficiencia respiratoria $(9,15,16)$.

Se investigó el beneficio potencial de utilizar una medida no invasiva de oxigenación para calcularla como una alternativa a la medición invasiva estándar, representada por el índice de mortalidad pediátrica, y se halló que los valores derivados se asociaron de forma significativa con la mortalidad en la unidad de cuidado intensivo, y que cuando fueron utilizadas todas las mediciones de $\mathrm{SpO}_{2} / \mathrm{FiO}_{2}$ disponibles, los valores derivados, como un único predictor, discriminaron 
moderadamente entre muerte y supervivencia en un subgrupo de pacientes (17). Los hallazgos realizados por Slater et al. (10), Bravo et al. (18) y Hammond et al. (19) generaron el análisis que permitió evaluar la validez del uso de mediciones no invasivas para la predicción de mortalidad. El hallazgo fue una asociación significativa entre una variable derivada de la relación $\mathrm{SpO}_{2} / \mathrm{FiO}_{2}$ y la mortalidad en todos los niños ingresados a la unidad de cuidado intensivo.

La puntuación del riesgo de mortalidad pediátrica evidencia una relación estadísticamente significativa entre los valores promedio, lo que constituye una valiosa información sobre el estado actual, la evolución del paciente y, eventualmente, la relación entre su riesgo y el nivel de tratamiento empleado. Palanca Arias (20), Lobeto Prieto et al. (21) y Murcia Sánchez (22) determinaron que los pacientes sometidos a ventilación mecánica que fallecieron tuvieron un índice de oxigenación alto, lo cual vaticina que predice la mortalidad al igual que la relación $\mathrm{PaO}_{2} / \mathrm{FiO}_{2}$ y confirma lo planteado por la literatura revisada. Esto coincide con lo descrito por Karbing et al. (23), quienes plantean que el índice de Kirby es un método adecuado para evaluar la oxigenación pulmonar en pacientes con insuficiencia respiratoria, válido además por Seeley et al. (24) y Tawfik et al. (25), como una medida del intercambio anormal de gases. En la actualidad, múltiples autores lo utilizan como un parámetro para evaluar el estado de oxigenación $y$, de esta forma, optimizar el soporte ventilatorio mecánico más adecuado de acuerdo con el trastorno presente en el paciente pediátrico.

Kim et al. (26) tomaron los índices de oxigenación y compararon la reclasificación basada en los datos medidos 24 horas después del diagnóstico con la clasificación inicial de síndrome de dificultad respiratoria, lo que permitió evidenciar un progreso en las primeras 24 horas, con respuesta adecuada de oxigenación y medida sustitutiva más discernible para establecer mortalidad. Ello fue similar a los resultados del estudio realizado por Segredo et al. (11), donde el índice de oxigenación y el de Kirby se constituyeron en predictores muy sensibles de pronóstico en los pacientes pediátricos ventilados.

A propósito de los estudios analizados en el contexto pediátrico, algunas investigaciones permitieron establecer una correlación entre la S/F y P/F ratio, con el fin de encontrar un marcador no invasivo alternativo utilizado para el diagnóstico y manejo del SDRA. Tales estudios, en su mayoría, concluyeron que la relación S/F es un marcador no invasivo confiable y continuamente disponible para evaluar el estado de oxigenación y diagnosticar lesión pulmonar aguda o SDRA en pacientes pediátricos. La relación $\mathrm{SpO}_{2} / \mathrm{FiO}_{2}$ tiene una asociación lineal con la $\mathrm{PaO}_{2} / \mathrm{FiO}_{2}$, siendo la $\mathrm{SpO}_{2} / \mathrm{FiO}_{2}$ un marcador no invasivo que puede sustituir a la relación $\mathrm{PaO}_{2} / \mathrm{FiO}_{2}$ en pacientes con insuficiencia respiratoria aguda hipoxémica (27-33). 


\section{Utilidad de la $\mathrm{PaO}_{2} / \mathrm{FiO}_{2}$ y $\mathrm{SpO}_{2} / \mathrm{FiO}_{2}$ en pacientes adultos en cuidado intensivo}

La relación $\mathrm{PaO}_{2} / \mathrm{FiO}_{2}$ es una herramienta utilizada en las unidades de cuidado intensivo adulto como un índice de oxigenación que, de forma invasiva, por medio de gases arteriales genera un diagnóstico y pronóstico de lesión pulmonar o SDRA. A partir del 2012 se postuló como uno de los criterios en la Definición de Berlín sobre la base de la hipoxemia, donde se clasifica el síndrome de dificultad respiratoria en leve $\left(\mathrm{PaO}_{2} / \mathrm{FiO}_{2}\right.$ de $200 \mathrm{a}$ $300 \mathrm{mmHg})$, moderado $\left(\mathrm{PaO}_{2} / \mathrm{FiO}_{2}\right.$ de 100 a 200 $\mathrm{mmHg})$ o severo $\left(\mathrm{PaO}_{2} / \mathrm{FiO}_{2}\right.$ menor de $100 \mathrm{~mm}$ Hg) (34-36).

En el estudio de Martínez et al. (37) se evaluó el rendimiento de los índices de oxigenación $\mathrm{PaO}_{2} /$ $\mathrm{FiO}_{2}$ y diferencia alveoloarterial de oxígeno, como predictores independientes de ventilación mecánica en pacientes con neumonía a 2600 metros de altitud. El resultado fue una adecuada aproximación para predecir la necesidad de ventilación mecánica en las primeras 72 horas, cuando el valor de $\mathrm{PaO}_{2} / \mathrm{FiO}_{2}$ era menor de $180 \mathrm{mmHg}(38-40)$.

Por otra parte, existe el índice conocido como $\mathrm{SpO}_{2} / \mathrm{FiO}_{2}$, utilizado como método continuo y no invasivo que, por medio de la oximetría de pulso, se convierte en una alternativa de monitorización respiratoria y valoración de la oxigenación del paciente críticamente enfermo. Desde el 2007,
Rice et al. (41) publicaron la correlación existente entre el índice $\mathrm{PaO}_{2} / \mathrm{FiO}_{2}$ y $\mathrm{SpO}_{2} / \mathrm{FiO}_{2}$ en paciente adultos con lesión pulmonar o SDRA, pues es útil tanto para el diagnóstico como para el seguimiento de este tipo de pacientes, pero con la necesidad de realizar nuevas investigaciones en poblaciones heterogéneas para validar la relación $(42,43)$.

El índice $\mathrm{SpO}_{2} / \mathrm{FiO}_{2}$ se toma como punto de partida teniendo en cuenta las limitaciones existentes en diferentes servicios de salud y regiones geográficas, en cuanto a la inaccesibilidad de gases arteriales, radiografía de tórax, entre otras pruebas, el cual fue desarrollado en el 2016 en Kigali (Ruanda), donde se crearon nuevos criterios que modificaron el uso de gases arteriales por la oximetría de pulso y permitieron sustituir la $\mathrm{PaO}_{2} / \mathrm{FiO}_{2}$ por el índice $\mathrm{SpO}_{2} / \mathrm{FiO}_{2}$, teniendo como base un valor menor o igual a $315 \mathrm{mmHg}$ para la clasificación de la severidad y, a su vez, disminuir la subestimación y subtratamiento en los países en desarrollo, según las diferentes disponibilidades y los recursos en la unidad de cuidado intensivo (44-47).

En el estudio de Pandharipande et al. (48) se validó el índice $\mathrm{SpO}_{2} / \mathrm{FiO}_{2}$ para la escala Sequential Organ Failure Assessment. Un objetivo de la investigación fue incluir el índice $\mathrm{SpO}_{2} / \mathrm{FiO}_{2}$ en lugar del $\mathrm{PaO}_{2} / \mathrm{FiO}_{2}$. Los autores concluyeron que los resultados obtenidos predicen resultados similares 
si no hay disponibilidad de gases arteriales, con puntuaciones de $\mathrm{SpO}_{2} / \mathrm{FiO}_{2}$ y $\mathrm{PaO}_{2} / \mathrm{FiO}_{2}$ altamente correlacionadas con una $p<0,001$ estadísticamente significativa, y respecto a la evaluación respiratoria de la Sequential Organ Failure Assessment, con un Spearman de 0,5, considerado correlación positiva de moderada a fuerte $(49,50)$.

Otro de los usos del índice $\mathrm{SpO}_{2} / \mathrm{FiO}_{2}$, incluidos en el estudio de Jaimes et al. (51), es como herramienta para predecir el requerimiento de ventilación mecánica en pacientes con neumonía adquirida en la comunidad. Allí se concluyó que este índice puede ser de gran utilidad para definir el uso de ventilación mecánica en los primeros 5 días, con un área bajo la curva (ROC) de 0,831 y un intervalo de confianza de $0,770-0,892$, en comparación con el ROC de 0,721 para la $\mathrm{PaO}_{2} /$ $\mathrm{FiO}_{2}(52-54)$.

Los estudios en adultos que comparan la correlación $\mathrm{PaO}_{2} / \mathrm{FiO}_{2}$ y $\mathrm{SpO}_{2} / \mathrm{FiO}_{2}$ han demostrado una correlación lineal, donde la $\mathrm{SpO}_{2} / \mathrm{FiO}_{2}$ podría ser sustituta de la $\mathrm{PaO}_{2} / \mathrm{FiO}_{2}$, con una sensibilidad y especificidad por encima del $80 \%$ en la mayoría de las investigaciones, que permite una identificación rápida en tiempo real como predictor temprano de desarrollo de SDRA, al igual que disminución de costos y complicaciones asociados a la toma de la prueba invasiva y su procesamiento $(4,7,55$ 57).

\section{$\mathrm{PaO}_{2} / \mathrm{FiO}_{2}$ y $\mathrm{SpO}_{2} / \mathrm{FiO}_{2}$ como predictores de mortalidad en cuidado intensivo adulto y pediátrico}

La insuficiencia respiratoria aguda que requiere soporte ventilatorio es una de las principales causas de mortalidad en las unidades de cuidado intensivo; por ello, los índices $\mathrm{PaO}_{2} / \mathrm{FiO}_{2}$ y $\mathrm{SpO}_{2} /$ $\mathrm{FiO}_{2}$ son marcadores de gravedad tanto en pacientes adultos como en niños críticamente enfermos (28). Diferentes investigaciones han concluido que los índices de oxigenación invasivos y no invasivos ofrecen capacidad predictiva moderada respecto a la mortalidad, con un rendimiento pronóstico similar para SDRA de moderado a severo (58-60).

En el estudio de cohorte observacional retrospectivo de Adams et al. (61) se incluyeron 23 hospitales, con una población de 28.758 ingresos, que generaron como resultado que por cada aumento del $10 \%$ en el tiempo en el riesgo determinado por la $\mathrm{SpO}_{2} / \mathrm{FiO}_{2}$, se asoció con un aumento del $24 \%$ en las probabilidades de mortalidad hospitalaria en pacientes sometidos a ventilación mecánica.

Para el estudio de Lai et al. (62) se concluye que una relación de la $\mathrm{PaO}_{2} / \mathrm{FiO}_{2}$ en el día cero no es un buen predictor de resultados; pero a las 24 horas posteriores al uso de ventilación mecánica, el índice es capaz de predecir con exactitud resultados en pacientes con SDRA. 
En el área de la pediatría se han realizado cálculos para los índices de mortalidad basados en los valores de saturación parcial de oxígeno y han concluido que la $\mathrm{SpO}_{2} / \mathrm{FiO}_{2}$ es útil y no presenta diferencias significativas respecto a los valores hallados de $\mathrm{PaO}_{2} / \mathrm{FiO}_{2}$ de forma invasiva por medio de la toma de gases arteriales. Sin embargo, las investigaciones mencionan que estas estimaciones deben confirmarse por medio de estudios multicéntrico-prospectivos más amplios (63-67).

A partir de los resultados se puede evidenciar que estos índices se aplican en el contexto clínico, tanto en pacientes pediátricos como en adultos; así mismo, que los usos de estos índices permiten evaluar la oxigenación en los pacientes con insuficiencia respiratoria y, de esta manera, establecer el manejo terapéutico indicado a partir del cuidado respiratorio.

Como limitante del estudio, se evidenció que para poder acceder a la información de algunos artículos en ciertas bases de datos existía restricción con necesidad de pago de acceso para visualizar todo el texto.

\section{CONCLUSIONES}

Los índices de oxigenación en el contexto clínico son fundamentales para evaluar la oxigenación de los pacientes con insuficiencia respiratoria y ha aumentado su uso en el cuidado intensivo pediátrico y adulto, dada la validez alternativa que han generado en el manejo terapéutico de los pacientes en estado crítico. Por otra parte, los resultados permiten dar cuenta de que la mayoría de los estudios elaborados desde el enfoque de utilidad de índices de oxigenación se caracterizan por una visión clínica.

De acuerdo con la revisión, el índice de saturación $\mathrm{SaO}_{2} / \mathrm{FiO}_{2}$ como marcador de oxigenación no invasivo podría usarse como sustituto en la evaluación de la oxigenación del paciente en estado crítico, ya que es una medida indirecta de la prueba GOLD de oxigenación, que corresponde a la $\mathrm{PaO}_{2} / \mathrm{FiO}_{2}$. Frente a la gravedad, estos índices permiten evaluar la severidad de la disfunción pulmonar en insuficiencia respiratoria, además de ser un marcador pronóstico de mortalidad tanto en pacientes adultos como en pediátricos.

Los resultados de la presente revisión pueden aportar al desarrollo de estudios clínicos que fundamenten la predicción que estos índices generan en los pacientes adultos y pediátricos en cuidado intensivo.

\section{CONFLICTO DE INTERESES}

Los investigadores declaran no presentar conflictos de intereses. 


\section{FINANCIAMIENTO}

Este estudio fue financiado por el Centro de Investigaciones para el Desarrollo (Cipade) de la Universidad de Boyacá y la ESE Hospital Universitario San Rafael.

\section{REFERENCIAS}

1. Arnedillo Muñoz A, García Polo C, García Jiménez JD. Valoración del paciente con insuficiencia respiratoria aguda y crónica. En: Soto Campos, coordinador. Manual de diagnóstico y terapéutica en neumología. 2 . $^{a}$ ed.; Madrid: Ggón; 2010. p. 225-32.

2. Gómez A, Aduen J, González JM, Moreno H, Fernández G, Quitián Ramírez D. Evaluación de la función de oxigenación pulmonar: análisis comparativo de 6 índices descritos en la literatura. Rev Colomb Anestesiol. 1990;18(2):119-26.

3. Adams JY, Rogers AJ, Schuler A, Marelich GP, Fresco JM, Taylor SL, et al. Association between peripheral blood oxygen saturation $\left(\mathrm{SpO}_{2}\right) /$ fraction of inspired oxygen $\left(\mathrm{FiO}_{2}\right)$ ratio time at risk and hospital mortality in mechanically ventilated patients. Perm J. 2020; 24:19.113. https://doi.org/10.7812/TPP/19.113
4. Ameghino Bautista J, Morales Corbacho J, Apolaya-Segura $\mathrm{M}$. Correlación entre $\mathrm{SO}_{2} /$ $\mathrm{FiO}_{2}$ y $\mathrm{PaO}_{2} / \mathrm{FiO}_{2}$ en pacientes con insuficiencia respiratoria en ventilación mecánica. Rev Cubana Inv Bioméd [internet]. 2019;37(3). Disponible en: http://www.revibiomedica.sld. cu/index.php/ibi/article/view/124

5. Mouret Hernández UEG, Mendoza Rodríguez M, López González A, Cortés Munguia A. Comparación de criterios de Berlín vs Kigali para diagnóstico del síndrome de insuficiencia respiratoria aguda. Med Crit [internet]. 2019;33(5):221-32. Disponible en: https:// www.medigraphic.com/pdfs/medcri/ti-2019/ ti195b.pdf

6. Chen WL, Lin WT, Kung SC, Lai CC, Chao CM. The value of oxygenation saturation index in predicting the outcomes of patients with acute respiratory distress syndrome. J Clin Med. 2018;7(8):205. https://doi.org/10.3390/ jcm7080205

7. Camajá HM, Ranero JL. Correlación entre los Índices $\mathrm{PaO}_{2} / \mathrm{FiO}_{2}$ y $\mathrm{SpO}_{2} / \mathrm{FiO}_{2}$ en pacientes con síndrome de distrés respiratorio en ventilación mecánica asistida. Rev Med Interna Guatem [internet]. 2017;21(1):135. Disponible en: http://docs.bvsalud.org/ biblioref/2019/06/995107/02.pdf 
8. Brown SM, Duggal A, Hou PC, Tidswell M, Khan $A$, Exline $M$, et al. Nonlinear imputation of $\mathrm{PaO}_{2} / \mathrm{FIO}_{2}$ from $\mathrm{SpO}_{2} / \mathrm{FIO}_{2}$ among mechanically ventilated patients in the ICU: a prospective, observational study. Crit Care Med. 2017;45(8):1317-24. https://doi.org/10.1097/ CCM. 0000000000002514

9. DesPrez K, McNeil JB, Wang C, Bastarache JA, Shaver CM, Ware IB. Oxygenation saturation index predicts clinical Outcomes in ARDS. Chest 2017;152(6):1151-8. https://doi. org/10.1016/j.chest.2017.08.002

10. Slater A, Straney L, Alexander J, Schell D, Millar J, for the Australian and New Zealand Intensive Care Society Pediatric Study Group and Centre for Outcomes and Resource Evaluation. The effect of imputation of $\mathrm{PAO}_{2} /$ $\mathrm{FiO}_{2}$ from $\mathrm{SPO}_{2} / \mathrm{FiO}_{2}$ on the performance of the Pediatric Index of Mortality. Pediatr Crit Care Med. 2020;21(6):520-5. https://10.1097/ PCC. 0000000000002233

11. Segredo Y, Alvera B, Acevedo Y, Rovira L, Álvarez I, Martell N. Índices de severidad respiratoria en el paciente pediátrico ventilado. Rev Cub Med Int Emerg [internet]. 2018;17(1):4759. Disponible en: http://www.revmie.sld.cu/ index.php/mie/article/view/185/html_136
12. Ameghino Bautista J, Morales Corbacho J, Apolaya-Segura $\mathrm{M}$. Correlación entre $\mathrm{SO}_{2} /$ $\mathrm{FiO}_{2}$ y $\mathrm{PaO}_{2} / \mathrm{FiO}_{2}$ en pacientes con insuficiencia respiratoria en ventilación mecánica. Rev Cubana Inv Bioméd [Internet]. 2018 [citado 2021 mar 12];37(3). Disponible en: http:// www.revibiomedica.sld.cu/index.php/ibi/ article/view/124

13. Gutiérrez Muñoz F. Ventilación mecánica. Acta Méd Peruana [Internet]. 2011 abr [citado 2021 mar 12];28(2):87-104. Disponible en: http://www.scielo.org.pe/ scielo.php? script $=$ sci_arttext\&pid $=$ S1728$59172011000200006 \&$ Ing $=$ es

14. González Vélez CL. Correlación $\mathrm{SO}_{2} / \mathrm{FiO}_{2}$ con $\mathrm{PaO}_{2} / \mathrm{FiO}_{2}$ en niños en ventilación mecánica a grandes alturas: estudio multicéntrico [internet]. Bogotá: Universidad del Rosario; 2012. Disponible en: http://repository.urosario. edu.co/handle/10336/3744

15. Valero Ortiz A, Umbacía Salas F, Palencia Mojica C, Suárez Saavedra M, Silva Rodríguez L. Uso de cánula nasal de alto flujo en falla respiratoria en adultos. Rev Investig Salud Univ Boyacá. 2019;6(1):170-87. https://doi. org/10.24267/23897325.406

16. Wilsterman $M E$, de Jager $P$, Blokpoel $R$, Frerichs I, Dijkstra SK, Albers MJIJ, et al. Short-term 
effects of neuromuscular blockade on global and regional lung mechanics, oxygenation and ventilation in pediatric acute hypoxemic respiratory failure. Ann Int Care. 2016;6:103. https://doi.org/10.1186/s13613-016-0206-9

17. Laila D, Yoel C, Hakimi H, Lubis M. Comparison of $\mathrm{SpO}_{2} / \mathrm{FiO}_{2}$ and $\mathrm{PaO}_{2} / \mathrm{FiO}_{2}$ ratios as markers of acute lung injury. Pediatric Indones. 2017;57(1):30-34. https://doi.org/10.14238/ pi57.1.2017.30-4

18. Bravo DL, López CF, Guzmán MC, Cely $\mathrm{JL}$, Izquierdo L. Predictors of successful extubation in a pediatric intensive care unit. Scientia Ricerca [internet]. 2017;1(2):113-20. Disponible en: https://scientiaricerca.com/ srprrc/pdf/SRPRRC-01-00014.pdf

19. Hammond $B$, García-Filion $P$, Kang $P$, Rao MY, Willis BC, Dalton HJ. Identifying an oxygenation index threshold for increased mortality in acute respiratory failure. Respir Care. 2017;62(10):1249-54. https://doi. org/10.4187/respcare.05092

20. Palanca Arias D. Valor predictor del cociente saturación hemoglobina/ $/ \mathrm{FiO}_{2}$ en pacientes pediátricos con insuficiencia respiratoria aguda debida a neumonía tratados con ventilación no invasiva [tesis doctoral en internet]. Santander (España): Departamento de Pediatría Hospital Universitario Marqués de Valdecilla; 2014. Disponible en: https://pdfs. semanticscholar.org/e3e4/b2fd0204ee8bf1b3 0d074ca4da07fe5a0bba.pdf

21. Lobeto Prieto C, Medina Villanueva A, Modesto V, Rey Galán C, Mayordomo Colunga J, Arcos Solas M. Predicción del índice $\mathrm{PaO}_{2} /$ $\mathrm{FiO}_{2}$ a partir del índice $\mathrm{SpO}_{2} / \mathrm{FiO}_{2}$ ajustado por la medición transcutánea de $\mathrm{COm}$ en niños críticamente enfermos. An Pediatr (Barc). 2011;74(2):91-6. https://doi.org/10.1016/j. anpedi.2010.09.021

22. Murcia Sánchez HE. Estudio de correlación entre la $\mathrm{PaO}_{2} / \mathrm{FiO}_{2}$ y la $\mathrm{SO} 2 / \mathrm{FiO}_{2}$ en niños en ventilación mecánica de la Fundación Cardioinfantil en Bogotá entre abril y junio de 2011 [tesis de especialización en internet]. Bogotá: Universidad del Rosario; 2011. Disponible en: https://core.ac.uk/download/ pdf/86437234.pdf

23. Karbing DS, Kjaergaard S, Smith B, Espersen $\mathrm{K}$, Allerod $\mathrm{C}$, Andreassen $\mathrm{S}$, Rees $\mathrm{S}$. Variation in the $\mathrm{PaO}_{2} / \mathrm{FiO}_{2}$ ratio with $\mathrm{FiO}_{2}$ : mathematical and experimental description, and clinical relevance. Crit Care. 2011;11:118. https://doi. org/10.1186/cc6174

24. Seeley E, McAuley DF, Eisner M, Miletin M, Matthay MA, Kallet RH. Predictors of mortality 
in acute lung injury during the era of lung protective ventilation. Thorax 2014;63:994-8. https://doi.org/10.1136/thx.2007.093658

25. Tawfik DS, Bennett TD, Welch B, Poss WB. Use of high-frequency ventilation in the pediatric intensive care unit. J Pediatr Intensive Care. 2016;5(1):12-20. https://doi. org/10.1055/s-0035-1568160

26. Kim SY, Kim B, Choi SH, Kim JD, Sol IS, Kim $\mathrm{MJ}$, et al. Oxygenation index in the first 24 hours after the diagnosis of acute respiratory distress syndrome as a surrogate metric for risk stratification in children. Acute Crit Care. 2018;33(4):222-9. https://doi.org/10.4266/ acc. 2018.00136

27. Laila $D$, Yoel $C$, Hakimi $H$, Lubis M. Comparison of $\mathrm{SpO}_{2} / \mathrm{FiO}_{2}$ and $\mathrm{PaO}_{2} / \mathrm{FiO}_{2}$ ratios as markers of acute lung injury. Paediatr Indones. 2017;57(1):30. https://doi.org/10.14238/ pi51.1.2011.30-4

28. Bilan N, Dastranji A, Ghalehgolab Behbahani A. Comparison of the $\mathrm{SpO}_{2} / \mathrm{FiO}_{2}$ ratio and the $\mathrm{PaO}_{2} / \mathrm{FiO}_{2}$ ratio in patients with acute lung injury or acute respiratory distress syndrome. J Cardiovasc Thorac Res. 2015;7(1):28-31. https://doi.org/10.15171/jcvtr.2014.06
29. Auliawati D, Suparyatha I, Wati D, Hartawan I, Subanada I. $\mathrm{SpO}_{2} / \mathrm{FiO}_{2}$ ratio as an oxygenation parameter in pediatric acute respiratory distress syndrome. Bali Med J. 2016;5(2):35861. https://doi.org/10.15562/bmj.v5i2.338

30. Lobete C, Medina A, Rey C, Mayordomo J, Concha $A$, Menéndez $S$. Correlation of oxygen saturation as measured by pulse oximetry/ fraction of inspired oxygen ratio with $\mathrm{PaO}_{2} /$ fraction of inspired oxygen ratio in a heterogeneous sample of critically ill children. J Crit Care. 2013;28(4):538.e1-7. https://doi. org/10.1016/j.jcrc.2012.12.006

31. Khemani RG, Thomas NJ, Venkatachalam V, Scimeme, JP, Berutti T, Schneider JB, et al. Comparison of $\mathrm{SpO}_{2}$ to $\mathrm{PaO}_{2}$ based markers of lung disease severity for children with acute lung injury. Crit Care Med. 2012;40(4):1309-16. https://doi.org/10.1097/ CCM.0b013e31823bc61b

32. Miranda MC, López-Herce J, Martínez MC, Carrillo A. Relación de la relación $\mathrm{PaO}_{2} / \mathrm{FiO}_{2}$ y $\mathrm{SatO}_{2} / \mathrm{FiO}_{2}$ con la mortalidad y la duración de ingreso en niños críticamente enfermos. An Pediatr. 2012;76(1):16-22. https://doi.org/ 10.1016/j.anpedi.2011.06.006

33. Leteurtre $S$, Dupré $M$, Dorkenoo A, Lampin ME, Leclerc F. Assessment of the Pediatric Index of 
Mortality 2 with the $\mathrm{PaO}_{2} / \mathrm{FiO}_{2}$ ratio derived from the $\mathrm{Spo}_{2} / \mathrm{FiO}_{2}$ ratio: a prospective pilot study in a French pediatric intensive care unit. Pediatr Crit Care Med. 2011;12(4):e184-6. https://doi. org/10.1097/PCC.0b013e3181fe3064

34. Sweeney RM, McAuley DF. Acute respiratory distress syndrome. Lancet. 2016;388(10058):2416-30. https://doi. org/10.1016/S0140-6736(16)00578-X

35. Lopes FM, Ferreira JR, Gusmao-Flores D. Impact of renal replacement therapy on the respiratory function of patients under mechanical ventilation. Rev Bras Ter Intensiva. 2013;25(3):251-7. https://doi. org/10.5935/0103-507X.20130044

36. Correa G. Caracterización de la función pulmonar en pacientes adultos despiertos en ventilación mecánica invasiva con diagnóstico de atelectasia, antes y después de ser reclutados con maniobra de insuflación sostenida. Cienc Salud [internet]. 2014;3(9):117. Disponible en: https://repository.usc. edu.co/bitstream/20.500.12421/853/1/ Caracterizaci \%C3 \%B3n \%20de \%20la \%20 funci \%C3 \%B3n \%20pulmonar \%20en \%20 pacientes $\% 20$ adultos $\% 20$ despiertos $\% 20$ en $\% 20$ ventilaci \%C3 \%B3n \% 20 mec \%C3\%A1 nica \%20invasiva.pdf
37. Martínez GM, Casas DP, Bastidas AR, Oliveros $H$, Pinilla PA, Calderón WJ, et al. Índices de oxigenación como predictores de ventilación mecánica en neumonía a 2600 metros de altitud. Acta Med Colomb [internet]. 2016;41(3). Disponible en: http://www.scielo. org.co/pdf/amc/v41n3/v41n3a06.pdf

38. Schmidt MF, Gernand J, Kakarala R. The use of the pulse oximetric saturation to fraction of inspired oxygen ratio in an automated acute respiratory distress syndrome screening tool. J Crit Care. 2015;30(3):486-90. https://doi. org/10.1016/j.jcrc.2015.02.007

39. Miyamoto $K$, Kawazoe $Y$, Yasuda $M$, et al. Oxygenation improves during the first $8 \mathrm{~h}$ of extended-duration prone positioning in patients with respiratory failure: a retrospective study. J Intensive Care. 2014;2:52. https://doi. org/10.1186/s40560-014-0052-5

40. Zhang F, Li C, Zhang J, Guo HP, Wu D-W. Comparison of quantitative computed tomography analysis and single-indicator thermodilution to measure pulmonary edema in patients with acute respiratory distress syndrome. BioMed Eng Online. 2014;13:30. https://doi.org/10.1186/1475-925X-13-30 
41. Rice T, Wheeler A, Bernard G, Schoenfeld D, Ware L. Comparison of the $\mathrm{SpO}_{2} / \mathrm{FiO}_{2}$ ratio and the $\mathrm{PaO}_{2} / \mathrm{FiO}_{2}$ ratio in patients with acute lung injury or ARDS. Chest. 2007;132:410-7. https://doi.org/10.1378/chest.07-0617

42. Villar J, Ambrós A, Soler JA, Martínez D, Ferrando $C$, Solano $R$, et al. Stratification and Outcome of Acute Respiratory Distress Syndrome (STANDARDS) network: age, $\mathrm{PaO}_{2} /$ $\mathrm{FiO}_{2}$, and plateau pressure score: a proposal for a simple outcome score in patients with the acute respiratory distress syndrome. Crit Care Med. 2016;44(7):1361-9. https://doi. org/10.1097/CCM.0000000000001653

43. Kangelaris KN, Ware LB, Wang CY, Janz DR, Zhuo $\mathrm{H}$, Matthay $M$, et al. Timing of intubation and clinical outcomes in adults with acute respiratory distress syndrome. Crit Care Med. 2016;44(1):120-9. https://doi.org/10.1097/ CCM.0000000000001359

44. Lazzeri C, Peris A. The Kigali modification of the berlin definition: ¿a new epidemiological tool for ARDS? J Thorac Dis. 2016;8(6):E443-5. https://doi.org/10.21037/jtd.2016.03.84

45. Riviello Ed, Kiviri $W$, twagirumugabe $t$, Mueller A, Banner-Goodspeed VM, officer $L$, et al. Hospital incidence and outcomes of ARdS using the Kigali modification of the
Berlin definition. Am J Respir Crit Care Med. 2016;193(1):52-9. https://doi.org/10.1164/ rccm.201503-05840C

46. Aslanidis Th, Myrou A, Tsirona Ch, Kontos A, Giannakou-Peftoulidou M. Comparison of $\mathrm{SpO}_{2} / \mathrm{FiO}_{2}$ ratio, oxygenation index, ventilator ratio and $\mathrm{SpO}_{2} / \mathrm{PaCO} 2$ ratio, $\mathrm{SpO}_{2} / \mathrm{PEEP}$ ratio with $\mathrm{PaO}_{2} / \mathrm{FiO}_{2}$ ratio in critically ill patients. The Greek E-Journal of Perioperative Medicine [internet]. 2016;14(a):36-44. Disponible en: http://e-journal.gr/wp/wp-content/uploads/ pdf/2016a/05.Comparison-of-SPO2-PACO2ratio-oxygenation-index.pdf

47. Festic E, Bansal V, Kor DJ, Gajic O; US Critical Illness and Injury Trials Group: Lung Injury Prevention Study Investigators (USCIITG-LIPS). $\mathrm{SpO}_{2} / \mathrm{FiO}_{2}$ ratio on hospital admission is an indicator of early acute respiratory distress syndrome development among patients at risk. J Intensive Care Med. 2015 May;30(4):209-16. https://doi.org/10.1177/0885066613516411

48. Pandharipande PP, Shintani AK, Hagerman $\mathrm{HE}$, St Jacques PJ, Rice TW, Sanders NW, et al. Derivation and validation of $\mathrm{SpO}_{2} / \mathrm{FiO}_{2}$ ratio to impute for $\mathrm{PaO}_{2} / \mathrm{FiO}_{2}$ ratio in the respiratory component of the Sequential Organ Failure Assessment score. Crit Care Med. 2009;37(4):1317-21. https://doi.org/10.1097/ cCM.0b013e31819cefa9 
49. Esteve F, López-Delgado JC, Javierre C, Skaltsa $\mathrm{K}$, Carrio MLL, Rodríguez-Castro $\mathrm{D}$, et al. Evaluation of the $\mathrm{PaO}_{2} / \mathrm{FiO}_{2}$ ratio after cardiac surgery as a predictor of outcome during hospital stay. BMC Anesthesiol. 2014;14:83. https://doi.org/10.1186/1471-2253-14-83

50. Aslanidis T, Myrou A, Chytas E, Anastasiou E, Geka $E$, Efthimiou $E$, et al. Relation between $\mathrm{PaO}_{2} / \mathrm{FiO}_{2}$ ratio, $\mathrm{SpO}_{2} / \mathrm{FiO}_{2}$ ratio, oxygenation index and ventilation ratio in critically ill patients. Crit Care. 2013;17(2):92. https://doi. org/10.1186/cc12030.

51. Jaimes $D$, Rengifo $D E$, Esneda L. $\mathrm{SaO}_{2} / \mathrm{FiO}_{2}$ tomada por pulso-oximetría, herramienta para predecir requerimiento de ventilación mecánica en pacientes con neumonía adquirida en la comunidad [tesis de especialización en internet]. Bogotá: Universidad Nueva Granada; 2014. Disponible en: http://hdl. handle.net/10654/12625

52. Festic E, Bansal V, J Kor D, Gajic O. $\mathrm{SpO}_{2} / \mathrm{FiO}_{2}$ Ratio on hospital admission is an indicator of early acute respiratory distress syndrome development among patients at risk. J Intensive Care Med. 2013;1-8. https://doi. org/10.1177/0885066613516411

53. Rincón Salas JJ. Correlación de los índices $\mathrm{PaO}_{2} / \mathrm{FiO}_{2}$ y $\mathrm{SpO}_{2} / \mathrm{FiO}_{2}$ en el postoperatorio de cirugía cardiaca. Rev Asoc Mex Med Crit y Ter Int [internet]. 2013;27(2):71-6. Disponible en: https://www.medigraphic.com/pdfs/medcri/ ti-2013/ti132b.pdf

54. Malicdem MG, Bnazon AG. Determination of the utility of the $\mathrm{SpO}_{2} / \mathrm{FiO}_{2}$ ratio in the diagnosis of patients admitted at the philippine heart center with acute lung injury or acute respiratory distress syndrome: a cross-sectional study. Chest. 2010;138(4):222. https://doi.org/10.1378/chest.10229

55. Yang $\mathrm{P}, \mathrm{Wu} \mathrm{T}, \mathrm{Yu} \mathrm{M}$, Chen F, Wang C, Yuan J, et al. A new method for identifying the acute respiratory distress syndrome disease based on noninvasive physiological parameters. PLoS One. 2020;15(2):e0226962. https://doi. org/10.1371/journal.pone.0226962

56. Bashar FR, Vahedian-Azimi A, Farzanegan B, Goharani R, Shojaei S, Hatamia S, et al. Comparison of non-invasive to invasive oxygenation ratios for diagnosing acute respiratory distress syndrome following coronary artery bypass graft surgery: a prospective derivation-validation cohort study. J Cardiothoracic Surg. 2018;13:123. https:// doi.org/10.1186/s13019-018-0804-8

57. Brown SM, Grissom CK, Moss M, Rice TW, Schoenfeld D, Hou PC, et al. Nonlinear 
Imputation of $\mathrm{PaO}_{2} / \mathrm{FiO}_{2}$ from $\mathrm{SpO}_{2} / \mathrm{FiO}_{2}$ among patients with acute respiratory distress syndrome. Chest. 2016;150(2):307-13. https:// doi.org/10.1016/j.chest.2016.01.003

58. Cedeño SL, Novillo JF. Índices de oxigenación y su relación con mortalidad en pacientes con síndrome de distress respiratorio agudo en unidades de cuidados intensivos de Quito [tesis de especialización en internet]. Quito: Pontifica Universidad Católica del Ecuador; 2019. Disponible en: http://repositorio. puce.edu.ec/bitstream/handle/22000/17205/ Tesis $\% 20 \%$ C $3 \%$ ADndices $\% 20$ de $\% 20$ oxigenaci \%C3 \%B3n \%20 en \%20SDRA. pdf? sequence $=1$ \&isAllowed $=y$

59. Macías MT, Muñoz MR, Cázarez CA. Índice de oxigenación como predictor de mortalidad en pacientes adultos con síndrome de insuficiencia respiratoria aguda en la unidad de cuidados intensivos. Rev Cub Med Int Emerg [internet]. 2012;11(4):2576-85. Disponible en: https://www.medigraphic.com/ pdfs/revcubmedinteme/cie-2012/cie124b.pdf

60. Rodríguez O, Rodríguez OB, Malberty J. Índice de oxigenación arterial en pacientes con sepsis respiratoria ventilados. MEDISAN [internet]. 2010; 4(2). Disponible en; http://scielo.sld. $\mathrm{cu} / \mathrm{scielo}$. php? script=sci_arttext\&pid=S102930192010000200013\&lng=es
61. Adams JY, Rogers AJ, Schuler A, Marelich G, Fresco J, Taylor $\mathrm{S}$, et al. Association between peripheral blood oxygen saturation $\left(\mathrm{SpO}_{2}\right)$ / fraction of inspired oxygen $\left(\mathrm{FiO}_{2}\right)$ ratio time at risk and hospital mortality in mechanically ventilated patients. Perm J. 2020;24:19.113. https://doi.org/10.7812/TPP/19.113

62. Lai CC, Sung MI, Liu HH, Chen CM, Chiang SR, Liu WL, et al. The ratio of partial pressure arterial oxygen and fraction of inspired oxygen 1 day after acute respiratory distress syndrome onset can predict the outcomes of involving patients. Medicine (Baltimore). 2016;95(14):e3333. https://doi.org/10.1097/ MD.0000000000003333

63. Ray S, Rogers L, Pagel C, Raman S, Peters $M$, Ramnarayan $\mathrm{P} . \mathrm{PaO}_{2} / \mathrm{FiO}_{2}$ ratio derived from the $\mathrm{SpO}_{2} / \mathrm{FiO}_{2}$ ratio to improve mortality prediction using the pediatric index of mortality-3 score in transported intensive care admissions. Pediatr Crit Care Med. 2017;18(3):e131-6. https://doi. org/10.1097/PCC.0000000000001075

64. Leteurtre $S$, Dupré $M$, Dorkenoo $A$, Lampin $M E$, Leclerc $F$. Assessment of the pediatric index of mortality 2 with the $\mathrm{PaO}_{2} / \mathrm{FiO}_{2}$ ratio derived from the $\mathrm{SpO}_{2} / \mathrm{FiO}_{2}$ ratio: a prospective pilot study in a French pediatric intensive care unit [published correction appears in Pediatr Crit Care Med. 2012 Jan;13(1):127]. Pediatr 
Crit Care Med. 2011;12(4):e184-6. https://doi. org/10.1097/PCC.0b013e3181fe3064

65. Feris Monterrosa N. Concordancia entre el índice $\mathrm{Sa} / \mathrm{Fi}$ y el índice $\mathrm{Pa} / \mathrm{Fi}$ para detección de hipoxemia en pacientes internados en unidad de cuidados intensivos [tesis de especialización en internet]. Cartagena de Indias: Universidad de Cartagena; 2017. http://repositorio. unicartagena.edu.co/bitstream/11227/5180/1/ Nazhid \%20Feris \%20Monterrosa.pdf

66. Shajin TA. Prospective observational study to compare the pulse oximetric saturation $\left(\mathrm{SpO}_{2}\right) /$ Fraction of Inspired Oxygen $\left(\mathrm{FiO}_{2}\right)$ (Sf ratio) and partial pressure of oxygen $\left(\mathrm{PaO}_{2}\right) / \mathrm{FiO}_{2}(\mathrm{PF}$ ratio) among critically ill children requiring respiratory support in a paediatric intensive care unit [Master of Medicine in Paediatric]. Vellore: Department of Paediatric Christian Medical College; 2017. http://repositorytnmgrmu.ac.in/4624/7/200701117shajin.pdf

67. Mantilla Alvarado JE. Correlación de los índices $\mathrm{PaO}_{2} / \mathrm{FiO}_{2}$ y SatO2$/ \mathrm{FiO}_{2}$ en pacientes adultos en oxigenoterapia [tesis bachiller en Medicina en internet]. Trujillo (Perú): Universidad Nacional de Trujillo; 2015. Disponible en: http://dspace. unitru.edu.pe/bitstream/handle/UNITRU/1113/ JUNNIOR \%20ENRIQUE \%20MANTILLA \%20 ALVARADO.pdf? sequence $=1$ \&isAllowed $=y$ 